\title{
Calabi-Yau Hypersurfaces in Products of Semi-Ample Surfaces
}

\author{
Paul Green ${ }^{1}$ and Tristan Hübsch ${ }^{2}$ \\ ${ }^{1}$ Department of Mathematics, University of Maryland, College Park, MD 20742, USA \\ ${ }^{2}$ Department of Physics and Astronomy, University of Maryland, College Park, MD 20742, \\ USA
}

\begin{abstract}
We study Calabi-Yau manifolds that are embedded as hypersurfaces in products of semi-ample complex surfaces. We classify the deformation classes of the latter and thereby achieve a classification of the Calabi-Yau manifolds that are constructed in this way. Complementing the results in the existing literature, we obtain the complete Hodge diamond for all Calabi-Yau hypersurfaces in products of semi-ample surfaces.
\end{abstract}

\section{Introduction}

In order to explore the phenomenological implications of the Superstring theories [1], originally defined in $9+1$ space-time dimensions, it was shown to be consistent to assume that the $9+1$-dimensional space-time locally has the form of $M_{4} \times \mathscr{M}_{\mathrm{CY}}$, where $M_{4}$ is $3+1$-dimensional Minkowski space and $\mathscr{M}_{\mathrm{CY}}$ is a CalabiYau manifold of 3 complex dimensions [2,3]. Calabi-Yau manifolds are compact and admit a Ricci-flat Kähler metric, i.e. have a vanishing first Chern class [4].

Several examples of such manifolds were analyzed in $[2,5,6]$. Upon restriction to massless states and compactification on a $\mathscr{M}_{\mathrm{CY}}$, the effective models exhibit $N=1$ supergravity and Yang-Mills interactions with the gauge group being a subgroup of $E_{6} \times E_{8}$, coupled to matter superfields the spectrum of which is counted by topological invariants of $\mathscr{M}_{\mathrm{CY}}$. In particular, superfields that transform as $(\mathbf{2 7}, \mathbf{1})$ of $E_{6} \times E_{8}$ are counted by the Hodge number $b_{1,2}$ while the $\left(27^{*}, \mathbf{1}\right)$ transforming superfields are $b_{1,1}$-fold degenerate.

In $[7,8]$ a huge family of Calabi-Yau manifolds was established all of which are embedded in products of complex-projective spaces as complete intersections of hypersurfaces (we shall adopt the CICY acronym [9] in what follows). Because of the fact that the Euler characteristic $\chi_{E}=2\left(b_{1,1}-b_{1,2}\right)$ for all Calabi-Yau manifolds, and since $\chi_{E}$ is computed straightforwardly for every case, the difference of the number of 27's and 27*'s of $E_{6}$ is readily obtained for models based on any of these manifolds. In contrast, the computation of $b_{1,2}$ and $b_{1,1}$ separately appears to be a much harder task. 
One method for computing $b_{1,1}$ relies on the Lefschetz Hyperplane Theorem (LHT) [10] applying it iteratively, as described in [8]. There we were able to prove the applicability of LHT only for a fairly restricted subset of CICY.

On the other hand, a different method was suggested in the literature $[3,11]$, presented as based on the deformation theory of Kodaira and Spencer [12]. In [13] we analyzed this method, proved that the argument generally given in support of these computations is seriously misleading and have given the correct proof of its applicability to the cases we found in the literature. Moreover, using the technique of spectral sequences we presented a general analysis which applies to all CICYs.

Our method, which is based on spectral sequences, yields sufficient conditions for the validity of the method of polynomial deformations, provides a computation of all Hodge numbers independently of any other, and applies to many cases where other methods fail. It also provides some information on the structure of the various cohomology groups on CICYs, so that an explicit construction generalizing that of the polynomial deformations method may be possible.

As we mentioned in [13], certain CICYs for which our method fails to determine $b_{1,1}$ and $b_{1,2}$ can be embedded as hypersurfaces in products of certain complex surfaces. Furthermore, for some of these manifolds LHT cannot be applied directly in the sense of [8].

It is the main goal of this paper to study the Calabi-Yau hypersurfaces in products of such surfaces and attempt to classify them.

The material of this paper is organized as follows: In Sect. 2 we define a family of surfaces in products of which Calabi-Yau manifolds can be embedded as hypersurfaces. We discuss the properties of these surfaces as abstract complex surfaces, construct "moduli spaces" for them, and show that many of them (we conjecture all) are complete intersections of hypersurfaces in products of complex projective spaces. In Sect. 3 we obtain the Hodge diamond for all Calabi-Yau hypersurfaces in products of these surfaces.

\section{The Surfaces}

The family of Calabi-Yau manifolds considered in [7-9,13] consists of complete intersections of hypersurfaces in products of complex-projective spaces $\mathscr{W}=\prod_{r=1}^{m} C P_{r}^{n_{r}}$. These hypersurfaces are defined by the vanishing of generic sets of holomorphic polynomials (hereafter constraints) such that the $a^{\text {th }}$ constraint is homogeneous of degree $q_{a}^{r}$ in the homogeneous coordinates of $C P_{r}^{n_{r}}$. It is useful to note that one may consider a set of holomorphic polynomials as a section of the global vector bundle:

$$
\mathscr{E}:=\bigoplus_{a=1}^{h}\left[\bigotimes_{r=1}^{m}\left(\mathscr{H}_{r}\right)^{q_{a}^{\prime}}\right], \quad \sum_{r=1}^{m} q_{a}^{r} \geqq 2 \text { for all } a,
$$

where $\mathscr{H}_{r}$ denotes the Hopf bundle (i.e. the bundle the sections of which correspond to linear homogeneous polynomials) on $C P_{r}^{n_{r}}$. We restrict to constraints of total degree at least two, since linear constraints are trivial in a sense: they only reduce 
$C P^{n}$ to $C P^{n-1}$. It has been proven in [8] that $\mathscr{M}:=v^{-1}(0)$ is a non-singular submanifold of $\mathscr{W}$ for $v$ a generic section of $\mathscr{E}$. For $\mathscr{M}$ to be a Calabi-Yau manifold, we need to ensure the vanishing of the first Chern class [4], which amounts to:

$$
\sum_{a=1}^{h} q_{a}^{r}=n_{r}+1, \text { for all } r .
$$

The column-vector of dimensions of the $C P_{r}^{n_{r}}$ s $s$ in $\mathscr{W}$ together with the matrix $\mathbf{q}$, in the notation of the form $(n \| \mathbf{q})$, was termed the configuration matrix and to the equivalence class of configuration matrices under permutations of rows of $(n \| \mathbf{q})$ and columns of $\mathbf{q}$ we refer simply as a configuration. Furthermore, a configuration represents a certain (partial!) deformation class of manifolds to which, in turn, the manifold $\mathscr{M}$ belongs.

The Calabi-Yau manifolds which form the subject of this paper are represented by configurations of the form:

$$
\left(\begin{array}{c|ccc}
n_{\Gamma} & \mathbf{q}(\Gamma) & \mathbf{0} & Q \\
n_{\Gamma^{\prime}} & \mathbf{0} & \mathbf{q}\left(\Gamma^{\prime}\right) & Q^{\prime}
\end{array}\right)=\left(\begin{array}{c||c}
\Gamma & Q \\
\Gamma^{\prime} & Q^{\prime}
\end{array}\right)
$$

where $\Gamma:=\left(n_{\Gamma} \| \mathbf{q}(\Gamma)\right)$ and $\Gamma^{\prime}:=\left(n_{\Gamma^{\prime}} \| \mathbf{q}\left(\Gamma^{\prime}\right)\right)$ are configurations representing surfaces, $Q$ is a column-vector with $m_{\Gamma}$ entries and $\mathbf{q}(\Gamma)$ is an $m_{\Gamma} \times h_{\Gamma}$ matrix of degrees of homogeneity of the constraints that define the configuration-surfaces $\Gamma$. To satisfy Eq. (2.2), the following must hold:

$$
Q_{r}=n_{r}+1-\sum_{a=1}^{h_{\Gamma}} q_{a}^{r}(\Gamma) \geqq 0, \text { for all } r,
$$

and similarly for $\Gamma^{\prime}$. Since we consider only indecomposable configurations in the sense of [8], neither $Q$ nor $Q^{\prime}$ may be the 0 -vector.

\subsection{The Problem and its Transposition}

In order to approach a classification of the surfaces $\mathscr{S}$ belonging to $\Gamma$ in (2.3), let us consider first some of their numeric invariants. For every surface $\mathscr{S}$ the Euler characteristic can be computed straightforwardly $[7,8]$. Independently, using e.g. the spectral sequence technique, as presented in [13], one can verify that for every surface $\mathscr{S}$ belonging to $\Gamma$ satisfying Eq. (2.4):

$$
b_{0,0}(\mathscr{S})=1, \quad b_{1,0}(\mathscr{S})=b_{2,0}(\mathscr{S})=0 .
$$

In particular, $b_{1,0}(\Gamma)$ is called irregularity ${ }^{1}$ of $\Gamma$ and its vanishing renders $\Gamma$ regular.

Note that $\chi_{E}$ determines all the Hodge numbers:

$$
\int_{0}^{0} b_{1,1}^{1} 0 \Rightarrow b_{1,1}=\chi_{E}-2
$$

\footnotetext{
${ }^{1}$ Throughout our analysis we shall rely on standard definitions and results in the algebraic geometry of surfaces as presented in [14]
} 
The reader should be cautioned that unlike the situation in complex dimension 1, it is not in general the case that the surfaces with the same Euler characteristic are deformations of one another.

Using standard expressions for the Euler characteristic and the Hirzebruch signature [14]:

$$
\begin{gathered}
\chi_{E}(\mathscr{S})=C_{2}(\mathscr{S})=2+b_{1,1}(\mathscr{S}), \\
\tau_{H}(\mathscr{S})=\frac{1}{3}\left(C_{1}^{2}(\mathscr{S})-2 C_{2}(\mathscr{S})\right)=2-b_{1,1}(\mathscr{S}),
\end{gathered}
$$

with $C_{1}^{2}$ and $C_{2}$ denoting the Chern numbers, one readily finds that:

$$
C_{1}^{2}(\Gamma)+C_{2}(\Gamma)=12 .
$$

The latter equation is just the statement of the Noether formula [14] for the surfaces that satisfy Eq. (2.4) and will be helpful because of the relation to the canonical bundle:

$$
C_{1}^{2}(\mathscr{S})=\mathscr{K}_{\mathscr{S}} \cdot \mathscr{K}_{\mathscr{S}},
$$

where $\mathscr{K}_{\mathscr{S}}$ is the bundle of holomorphic 2-forms of $\mathscr{S}$.

$\mathscr{K}_{\mathscr{S}}^{*}$, the anti-canonical bundle, is the restriction to $\mathscr{S}$ of:

$$
\bigotimes_{r=1}^{m_{\Gamma}}\left(\mathscr{H}_{r}\right)^{Q_{r}}
$$

For the $Q_{r}$ 's as in Eq. (2.4), $\mathscr{K}_{\mathscr{S}}^{*}$ is non-trivial and, for each point of $\mathscr{S}$, has by Eq. (2.4) at least one section which does not vanish there. In particular, it follows that rank $H^{0}\left(\mathscr{S}, \mathscr{K}_{\mathscr{S}}^{*}\right) \geqq 2$. Consequently, the $g$-fold tensor-products of $\mathscr{K}_{\mathscr{S}}^{*}$, $\left(\left(\mathscr{K}_{\mathscr{S}}^{*}\right)^{g}\right)$, are non-trivial as well and have sections. Thus, it follows for the $g$-fold tensor-products of the canonical bundle:

$$
P_{g}:=H^{0}\left(\Gamma,\left(\mathscr{K}_{\mathscr{S}}\right)^{g}\right)=0,
$$

i.e. all the plurigenera vanish.

Comparing our situation with the existing classification of the complex surfaces we see that all the surfaces we are studying have Kodaira dimension $\kappa(\mathscr{S})=-1$ [by Eq. (2.11)]. Then, since $\Gamma$ are all regular algebraic surfaces, it follows by the Castelnuovo-Enriques theorem that they are rational. By the classification of rational surfaces every $\mathscr{S}$ is a $C P^{2}$ or $S_{n}$ with finitely many (possibly no) points blown-up. $S_{n}$ are the Hirzebruch (rational ruled) surfaces, all of which have $\chi_{E}=4$ and which are distinguished one from another by the fact that $S_{n}$ contains an exceptional line of the $n^{\text {th }} k i n d$, i.e. a line with self-intersection $-n, n \geqq 0$.

We shall shortly prove that for our purpose $S_{n}, n>2$ need not be considered and thus we need only consider possible blow-ups of $C P^{2}, S_{0}, S_{1}$, and $S_{2}$. Before doing so, we recall some standard facts in algebraic geometry:

Proposition 1. Let $\widetilde{\mathscr{S}}_{p}$ denote the surface obtained from $\mathscr{S}$ by blowing it up at $p$. Then the space of sections of the anti-canonical bundle, $\mathscr{K}_{\mathscr{\mathscr { T }}_{p}}^{*}$, may be identified with the space of sections of $\mathscr{K}_{\mathscr{Y}}^{*}$ that vanish at $p$.

Proof. Let $\left(z_{1}, z_{2}\right)$ be local coordinates on $\mathscr{S}$ at $p=(0,0)$. Let also $\left(\zeta_{1}:=z_{1}, \zeta_{2}:=z_{2} / z_{1}\right)$ whenever $z_{1} \neq 0\left(z_{1}=0\right.$ is easily covered by an analogous 
coordinate patch). Note that in the limit where $\zeta_{2}$ is constant and $z_{1}=\zeta_{1} \rightarrow 0, \zeta_{2}$ may be used to parametrize the $C P^{1}$ into which $p$ is blown up. We have:

$$
d z_{1} \wedge d z_{2}=\zeta_{1} \cdot d \zeta_{1} \wedge d \zeta_{2}
$$

and note that the left-hand side is a section of the canonical bundle $\mathscr{K}_{\mathscr{S}}$, while the right-hand side represents a section of the canonical bundle $\mathscr{K}_{\check{I}_{p}}$. Let $\sigma$ be a section of the anti-canonical bundle on $\mathscr{S} \backslash\{p\}=\tilde{\mathscr{S}}_{p} \backslash\left\{C P^{1}\right\}$. Then:

$$
\left\langle\sigma, d z_{1} \wedge d z_{2}\right\rangle=\zeta_{1}\left\langle\sigma, d \zeta_{1} \wedge d \zeta_{2}\right\rangle
$$

by the natural pairing between $\mathscr{K}$ and $\mathscr{K}^{*}$. Consequently, if $\sigma$ extends over $\widetilde{\mathscr{S}}_{p}$, we have

$$
\lim _{\substack{\zeta_{1} \rightarrow 0 \\ \zeta_{2} \text { fixed }}}\left\langle\sigma, d z_{1} \wedge d z_{2}\right\rangle=0
$$

independently of $\zeta_{2}$. Therefore,

$$
\lim _{\left(z_{1}, z_{2}\right) \rightarrow(0,0)}\left\langle\sigma, d z_{1} \wedge d z_{2}\right\rangle=0
$$

and $\sigma$ extends to $\mathscr{S}$, taking the value 0 at $p$.

Conversely, if $\sigma$ is a section of the anti-canonical bundle of $\mathscr{S}$, vanishing at $p$, we can extend $\sigma$ over $\tilde{\mathscr{S}}_{p}$ by setting:

$$
\left.\left\langle\sigma, d \zeta_{1} \wedge d \zeta_{2}\right\rangle\right|_{\text {at }\left(0, \zeta_{2}\right)}=\lim _{\substack{z_{1} \rightarrow 0 \\\left(z_{2} / z_{1}\right) \text { fixed }}} \frac{\left\langle\sigma, d z_{1} \wedge d z_{2}\right\rangle}{z_{1}} .
$$

Proposition 2. Let $D$ be a non-singular divisor on the surface $\mathscr{S}$ and $p \in D$. Let $\widetilde{\mathscr{S}}_{p}$ be $\mathscr{S}$ blown up at $p$ and $\widetilde{D}$ the proper transform of $D$. Then the self-intersection $\widetilde{D} \cdot \widetilde{D}$ $=D \cdot D-1$. (For a proof we refer to $[14$, p. 476$]$.)

Motivated by the considerations so far, we shall adopt the following definition:

Definition. A complex surface, $\mathscr{S}$, is called semi-ample if:

1. The canonical bundle, $\mathscr{K}_{\mathscr{S}}$ is non-trivial;

2. $\mathscr{K}_{\mathscr{S}}^{*}$ has at least one non-vanishing section at every point of $\mathscr{S}$.

This definition has the advantage of being based on the properties of the canonical bundle of $\mathscr{S}$, which is purely intrinsic to $\mathscr{S}$. It however does cover all the surfaces that belong to configuration surfaces $\Gamma$. As a simple corollary to the definition, it follows that $\mathscr{K}_{\mathscr{S}}^{*}$ of a semi-ample surface $\mathscr{S}$ has at least two sections. We shall hereafter refer to a semi-ample regular (hence rational) surface as a SAR.

Proposition 3. The anti-canonical bundle of a $\mathrm{SAR} \mathscr{S}$ is induced from the Hopf bundle, $\mathscr{H}$, by a map $\mathscr{S} \stackrel{\pi}{\longrightarrow} C P^{\eta-1}$, where $\eta$ is the number of linearly independent sections of $\mathscr{K}_{\mathscr{S}}^{*}$.

Proof. Let $V$ be the space of sections of $\mathscr{K}_{\mathscr{S}}^{*}$. Set $C P^{\eta-1}:=P\left(V^{*}\right)$. For $s \in \mathscr{S}$ set $V_{\mathscr{S}}=\{\sigma \in V \mid \sigma(s)=0\}$. Then $\pi(s)$ is the one-dimensional subspace of $V^{*}$ which is identified with $V / V_{\mathscr{S}}$ by the natural pairing, $V \otimes V^{*} \rightarrow \mathbf{C}$.

Proposition 4. A SAR has no line with self-intersection less than -2 . 
Proof. Assume that SAR $\mathscr{S}$ contains a line $L$ with self-intersection $L \cdot L=-n, n>0$. It is standard that $\mathscr{K}_{L}=T^{*}(L)=\mathscr{H}_{L}^{-2}$, i.e. $T(L)=\mathscr{H}_{L}^{2}$ (since $L \approx C P^{1}$ ). Since $L \cdot L=-n$, the normal bundle of $L$ is given by $N(L)=\mathscr{H}_{L}^{-n}$. But then the anticanonical bundle of $\mathscr{S}$ at $L$ is:

$$
\left.\mathscr{K}_{\mathscr{S}}^{*}\right|_{L}=T(L) \otimes N(L)=\mathscr{H}_{L}^{(2-n)} .
$$

Thus if $n>2, \mathscr{K}_{\mathscr{S}}^{*}$ would have no non-trivial non-vanishing sections along $L \subset \mathscr{S}$, contradicting the definition of a SAR.

Proposition 5. Every SAR is $S_{0}=C P^{1} \times C P^{1}, S_{2}$ or the result of a (possibly empty) sequence of successive blow-ups of $C P^{2}$. ( $S_{1}$ is a blow-up of $C P^{2}$ at one point.)

Proof. A generic point of $S_{n}$ is one that is not on the exceptional line; a non-generic point is one which is. $S_{n}$ blown up at a generic point is the same as the blow-up of $S_{n-1}$ at a non-generic point. It follows that any blow-up of $S_{2}$ at a non-generic point contains a line of self-intersection -3 , and that $S_{n}, n \geqq 3$ or any blow-up thereof contains a line of self-intersection $\leqq-3$. On the other hand, a blow-up of $S_{2}$ at a generic point can also be realized as a blow-up of $S_{1}$.

As in [14], we shall call $p_{2}$ infinitely near $p_{1}$ if $p_{2}$ is on the (exceptional) line created by blowing up $p_{1}$. Further, a sequence of points $\left\{p_{1}, \ldots, p_{n}\right\}$ we call a stack if $p_{i}$ is infinitely near $p_{i-1}$ for each $1<i \leqq n$.

Proposition 6. A blow-up $\widetilde{C P}_{p_{1}, \ldots, p_{n}}^{2}$ is a SAR only if:

1) No 2 of the points are infinitely near a $3^{\text {rd }}$;

2) No 4 of the points form a stack;

3) No 4 of the points are on (the proper transform of) a line in $C P^{2}$;

4) No 7 of the points are on (the proper transform of) a quadric in $C P^{2}$.

Proof. The violation of any of the conditions 1, 3, and 4, creates a line of selfintersection $\leqq-3$ by Proposition 2 , since:

$\left.1^{\prime}\right)$ the line created by blowing up a point has self-intersection -1 ;

$\left.3^{\prime}\right)$ a line in $C P^{2}$ has self-intersection 1 ;

$4^{\prime}$ ) a quadric in $C P^{2}$ has self-intersection 4.

Suppose now that $p_{1}, p_{2}, p_{3}$, and $p_{4}$ form a stack. Then the sections $\sigma$ of $\mathscr{K}_{\mathscr{S}}^{*}$ (which, we recall, are cubics) corresponding to sections of $\mathscr{K}^{*}\left(\widetilde{C P_{p_{1}}^{2}, \ldots, p_{4}}\right)$ satisfy:

i) $\sigma\left(p_{1}\right)=0$;

ii) $\sigma_{p_{2}}^{\prime}\left(p_{1}\right)=0$, where $\sigma_{p_{2}}^{\prime}\left(p_{1}\right)$ is the derivative of $\sigma$ [well defined because $\left.\sigma\left(p_{1}\right)=0\right]$ in a direction determined by $p_{2}$;

iii) $\sigma_{p_{2}, p_{3}}^{\prime \prime}\left(p_{1}\right)=0$, where $\sigma_{p_{2}, p_{3}}^{\prime \prime}\left(p_{1}\right)$, is a second derivative in directions determined by $p_{2}$ and $p_{3}$.

To show the necessity of 2 , we begin by recalling that sections of $\mathscr{K}_{C P^{2}}^{*}$ are homogeneous cubic polynomials. If we blow up $p_{1}$, the sections of the anticanonical bundle of $\widetilde{C P}_{p_{1}}^{2}$ correspond to cubic polynomials vanishing at $p_{1}$ and the values of such a cubic on the line created by blowing up $p_{1}$ are determined by the derivatives of the cubic at $p_{1}$ (see Proof of Proposition 1).

If $p_{2}$ is infinitesimally near $p_{1}$, in order to obtain a section of $\mathscr{K} *\left(\widetilde{C P_{p_{1}}^{2} p_{2}}\right)$, we require in addition that some derivative vanishes at $p_{1}$, and the values on the line created by blowing up $p_{2}$ are determined by certain second derivatives, which are 
well defined because of the conditions we have imposed so far. Similarly, blowing up $p_{3}$ infinitely near $p_{2}$ imposes a vanishing condition on some $2^{\text {nd }}$ derivative and the values along the new exceptional line are determined by $3^{\text {rd }}$ derivatives. If we blow up $p_{4}$ infinitely near $p_{3}$, the values of the sections of $\mathscr{K}^{*}\left(\widetilde{C P}_{p_{1}, \ldots, p_{4}}^{2}\right)$ along the line created by blowing up $p_{4}$ will be determined by $4^{\text {th }}$ derivatives, all of which vanish for a cubic. Hence, if $p_{1}, \ldots, p_{4}$ forms a stack, $\widetilde{C P_{p_{1}}^{2}, \ldots, p_{4}}$ is not a SAR.

Proposition 7. If $n \leqq 8$ and $p_{1}, \ldots, p_{n}$ satisfy 1, 2, 3, and 4 of Proposition 6, then $p_{1}, \ldots, p_{n}$ impose independent conditions on cubics, i.e. the space of cubics on $C P^{2}$ vanishing at $p_{1}, \ldots, p_{n}$ has dimension $10-n$.

Proof. The space of cubics on $C P^{2}$ is 10-dimensional. What is required is to show that if $p_{1}, \ldots, p_{n}$ satisfy the hypotheses there is a cubic curve which contains all but one of the points and not the remaining one. This can easily be done by a straightforward adaptation of the proof of the lemma on p. 481 of [14].

Corollary 1. No SAR has $\chi_{E}=11$.

Proof. By Proposition 5, a SAR with $\chi_{E}=11$ must be a successive blow-up of $C P^{2}$ at eight points. By Proposition 1 only those sections of $\mathscr{K}_{C P^{2}}^{*}$ extend to the blow-up that vanish at the points to be blown up. As by Proposition 7 only two of ten linearly independent sections of $\mathscr{K}_{C P^{2}}^{*}$ vanish at those eight points, they vanish at a ninth point as well, since two plane cubic curves meet in 9 points. Blowing up that point will increase $\chi_{E}$ to 12 , and if it is not blown up every section of the anticanonical bundle will vanish there, contradicting our definition of a SAR.

Proposition 8. Let $\mathscr{S}$ be a SAR with $3 \leqq \chi_{E} \leqq 10$. Then $H^{1}\left(\mathscr{S}, \mathscr{K}_{\mathscr{S}}^{*}\right)=0$.

Proof. Applying the Hirzebruch-Riemann-Roch formula ${ }^{2}$ (see e.g. $[15$, p. 5]) to $\mathscr{K}_{\mathscr{S}}^{*}$ :

$$
\sum_{i=0}^{2}(-1)^{i} \operatorname{rank} H^{i}\left(\mathscr{P}, \mathscr{K}_{\mathscr{S}}^{*}\right)=\frac{1}{2}\left[c_{1}^{2}\left(\mathscr{K}_{\mathscr{S}}^{*}\right)+c_{1}\left(\mathscr{K}_{\mathscr{S}}^{*}\right) \cdot c_{1}(\mathscr{S})\right]+\frac{1}{12}\left[c_{1}^{2}(\mathscr{S})+c_{2}(\mathscr{S})\right] .
$$

Note that using Eq. (2.7) and Eq. (2.8), the right-hand side of this equation becomes $13-\chi_{E}$, and that, by Serre duality,

$$
H^{2}\left(\mathscr{S}, \mathscr{K}_{\mathscr{S}}^{*}\right)=H^{0}\left(\mathscr{S}, \mathscr{K}_{\mathscr{Y}}^{2}\right)=0 .
$$

On the other hand, by Proposition $7, \operatorname{rank} H^{0}\left(\mathscr{S}, \mathscr{K}_{\mathscr{S}}^{*}\right)=13-\chi_{E}(\mathscr{S})$ for $3 \leqq \chi_{E}(\mathscr{S})$ $\leqq 10$, and the statement follows.

Remark. Note that the same argument implies that $\operatorname{rank} H^{1}\left(\mathscr{S}, \mathscr{K}_{\mathscr{S}}^{*}\right)=1$ for a SAR with $\chi_{E}=12$, since then $\operatorname{rank} H^{0}\left(\mathscr{S}, \mathscr{K}_{\mathscr{S}}^{*}\right)=2$.

Theorem 1. For every $\operatorname{SAR} \mathscr{S}$ which is an iterated blow-up of $C P^{2}$, the 0-set of a generic section of $\mathscr{K}_{\mathscr{S}}^{*}$ is the proper transform of a non-singular cubic. (This means that the set of sections for which the conclusion is true contains a dense open subset of the space of all sections.)

Proof. By Proposition 3 of this paper and Proposition 2 of [8], there exists a dense and open set $(\mathscr{U})$ of sections of $\mathscr{K}_{\mathscr{S}}^{*}$ such that their 0 -set is non-singular on $\mathscr{S}$.

\footnotetext{
${ }^{2}$ We are indebted to Jonathan Rosenberg for suggesting this argument
} 
By iteration of Proposition 1, the 0-set of any section of $\mathscr{K}_{\mathscr{S}}^{*}$ is the proper transform of a cubic. For all $\sigma \in \mathscr{U}$, the only possible singularity of $\sigma^{-1}(0)$ on $C P^{2}$ are points that have been blown up. For each $p$ blown up, a dense open subset $\left(\widetilde{\mathscr{U}}_{p}\right)$ of the sections of $\mathscr{K}_{\mathscr{S}}^{*}$ has non-vanishing one-jets at $p$ (otherwise $\mathscr{S}$ cannot be a SAR).

But $\mathscr{U} \cap \bigcap_{p_{\text {blown up }}} \tilde{\mathscr{U}}_{p}$ is dense and open.

Corollary 2. No SAR has $\chi_{E}>12$.

Proof. Suppose $\mathscr{S}$ were a SAR with $\chi_{E}>12$. By Theorem 1 there is a section $\sigma$ of $\mathscr{K}_{\mathscr{S}}^{*}$ such that $\sigma^{-1}(0)$ is the proper transform of a non-singular cubic curve on $C P^{2}$. Let $C$ and $\widetilde{C}$ be the cubic curve and its proper transform respectively. By Proposition 1, all the $\chi_{E}-3$ blown up points are on $C$. We have $C \cdot C=9$ (since they are generic cubics), and by Proposition $2, \widetilde{C} \cdot \widetilde{C}=12-\chi_{E}$. But $\widetilde{C} \cdot \widetilde{C}$ is also the Chern number of the $N(\widetilde{C})$, the normal bundle of $\widetilde{C}$ in $\mathscr{S}$ and, since the tangent bundle of $\widetilde{C}$ has vanishing Chern class, $\widetilde{C} \cdot \widetilde{C}$ is also the Chern number of $\left.\mathscr{K}_{\mathscr{S}}^{*}\right|_{\tilde{C}}$.

Hence if $\widetilde{C} \cdot \widetilde{C}<0$, all sections of $\mathscr{K}_{\mathscr{S}}^{*}$ vanish along $\widetilde{C}$ and therefore $\mathscr{S}$ is not a SAR.

\subsection{Positive SARs}

Many of the surfaces we are discussing share a property stronger than semiampleness. Let us therefore recall that [14]:

Definition. A line bundle $\mathscr{L} \rightarrow \mathscr{M}$ is positive if there exists a metric on $\mathscr{L}$ with curvature form $\Theta$ such that $(\sqrt{-1} / 2 \pi) \Theta$ is a positive $(1,1)$-form.

Definition. A SAR is positive if it has positive anti-canonical bundle.

Corollary 3. No positive SAR has $\chi_{E}>10$.

Proof. By repeating the arguments of the Proof of Corollary 2, we obtain that if $\chi_{E}(\mathscr{S}) \geqq 12, \widetilde{C} \cdot \widetilde{C} \leqq 0$ and $\mathscr{K}_{\mathscr{S}}^{*}$ is not positive.

Theorem 2. If $\mathscr{S}$ is a positive $\mathrm{SAR}, \mathscr{S}$ is one of the following:

1) $S_{0}=C P^{1} \times C P^{1}$;

2) $C P^{2}$ blown-up at a set of at most 7 points in general position:

- no point is infinitesimally near another (no stacks),

- no 3 points lie on a line,

- no 6 points lie on a quadric.

Proof. By Proposition 5, $\mathscr{S}$ is $C P^{1} \times C P^{1}$ or an iterated blow-up of $C P^{2}$. If at some iteration we blow up a point on the exceptional line created by a previous blow-up, we create an exceptional line of $2^{\text {nd }}$ kind. $\mathscr{K}_{\mathscr{S}}^{*}$ restricted to such a line is trivial and it follows that $\mathscr{K}_{\mathscr{S}}^{*}$ is not positive. Hence the points which are blown up have to be distinct points of $C P^{2}$.

Blowing up a point on any curve decreases its self-intersection by 1 , by Proposition 2.

Thus, straightforwardly, blowing-up 3 (6) points on a line (quadric) creates an exceptional line of the $2^{\text {nd }}$ kind and as above renders the surface non-positive. 
Recalling the discussions in Sect. 2, it is clear that every configuration surface is a SAR. While we conjecture the converse of this, we have not been able to prove it. The following two theorems, however, represent some progress in that direction.

Theorem 3. If $\mathscr{S}$ is a $S_{0}$ or a successive blow-up of $C P^{2}$ at up to 6 points in general position, it belongs to one of the configurations below.

Proof. We shall prove this case by case for $3 \leqq \chi_{E} \leqq 9$.

1. $S_{0}=C P^{1} \times C P^{1} \in(3 \| 2)_{4} \cong\left(\left.\begin{array}{l}1 \\ 1\end{array}\right|_{4}=: \Gamma_{4}^{0}\right.$ (see $[14$, p. 478$\left.]\right)$.

2. $C P^{2}$ belongs to a "trivial" configuration, $(2 \mid)=: \Gamma_{3}$, with no constraints.

3. $S_{1}=\widetilde{C P_{p_{1}}^{2}} \in\left(\begin{array}{l|l}2 & 1 \\ 1 & 1\end{array}\right)_{4}=: \Gamma_{4}^{1}$. To see this, note that the bi-linear constraint takes the general form.

$$
\mathscr{A}(2 \| 1) \cdot z_{0}+\mathscr{B}(2 \| 1) \cdot z_{1}=0, \quad\left(z_{0}, z_{1}\right) \in C P_{2}^{1},
$$

where $\mathscr{A}(2 \| 1)$ and $\mathscr{B}(2 \| 1)$, the "coefficients" in Eq. (2.12), are linear in $C P_{1}^{2}$. When both of these "coefficients" vanish, $\left(z_{0}, z_{1}\right)$ are unconstrained by Eq. (2.12) and span all of $C P_{2}^{1}$. This happens in a single point of $C P_{1}^{2}$, since $\mathscr{A}=0$ and $\mathscr{B}=0$ describe lines in $C P_{1}^{2}$, which meet only in a point $\left(\chi_{E}\left(\begin{array}{ll}2 \| 1 & 1\end{array}\right)=1\right)$. Therefore, this single point of $C P_{1}^{2}$ is blown up (into the $C P_{2}^{1}$ ) which is exactly the description of $S_{1}$. The converse follows by construction.

4. $\widetilde{C P}_{p_{1}, p_{2}}^{2} \in\left(\begin{array}{l|ll}2 & 1 & 1 \\ 1 & 1 & 0 \\ 1 & 0 & 1\end{array}\right)=: \Gamma_{5}$. The analysis of 3 is repeated twice.

5. The argument of 3 can be repeated straightforwardly three times for

$$
\widetilde{C P_{p_{1}, p_{2}, p_{3}}^{2}} \in\left(\begin{array}{l||lll}
2 & 1 & 1 & 1 \\
1 & 1 & 0 & 0 \\
1 & 0 & 1 & 0 \\
1 & 0 & 0 & 1
\end{array}\right) .
$$

If the three points are not collinear, then moreover:

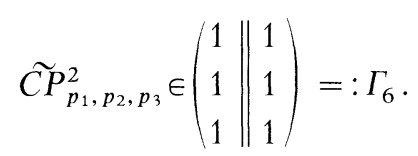

To prove this relation, consider the three bi-linear constraints in (2.13) as a system of three linear equations in $(x, y, z) \in C P^{2}$. For this system to have a solution at all $\left((0,0,0) \notin C P^{2}\right)$, we must have:

$$
\operatorname{det}\left(\begin{array}{ccc}
\mathscr{A}_{1} & \mathscr{B}_{1} & \mathscr{C}_{1} \\
\mathscr{A}_{2} & \mathscr{B}_{2} & \mathscr{C}_{2} \\
\mathscr{A}_{3} & \mathscr{B}_{3} & \mathscr{C}_{3}
\end{array}\right)=0
$$

where $\mathscr{A}_{i}, \mathscr{B}_{i}$, and $\mathscr{C}_{i}$ are linear in $C P_{i}^{1}$. Equation (2.14) is the candidate tri-linear constraint needed to embed $\widetilde{C P}_{p_{1}, p_{2}, p_{3}}^{2}$ in $C P^{1} \times C P^{1} \times C P^{1}$. For the hypersurface 
to be non-singular, the matrix in Eq. (2.14) must be of rank 2. For it to be of rank $\leqq 1$, in addition to Eq. (2.14), the sub-determinants:

$$
\operatorname{det}\left(\begin{array}{ll}
\mathscr{A}_{1} & \mathscr{B}_{1} \\
\mathscr{A}_{2} & \mathscr{B}_{2}
\end{array}\right), \quad \text { and } \operatorname{det}\left(\begin{array}{cc}
\mathscr{A}_{1} & \mathscr{C}_{1} \\
\mathscr{A}_{3} & \mathscr{C}_{3}
\end{array}\right), \quad \text { and } \operatorname{det}\left(\begin{array}{cc}
\mathscr{B}_{2} & \mathscr{C}_{2} \\
\mathscr{B}_{3} & \mathscr{C}_{3}
\end{array}\right)
$$

ought to vanish as well. Let $p_{1}, p_{2}$, and $p_{3}$ be the three points to be blown up. Let us choose homogeneous coordinates $(x, y, z)$ on $C P^{2}$ so that the line $l_{x=0}=\overline{p_{1} p_{2}}$, $l_{y=0}=\overline{p_{1} p_{3}}$, and $l_{z=0}=\overline{p_{2} p_{3}}$.

Then we may write the homogeneous polynomials as:

$$
\begin{aligned}
\mathscr{A}_{1} x+\mathscr{B}_{1} y \quad & =0, \\
\mathscr{A}_{2} x+\quad \mathscr{C}_{2} z & =0, \\
\mathscr{B}_{3} y+\mathscr{C}_{3} z & =0,
\end{aligned}
$$

where the "coefficients" in the $i^{\text {th }}$ equation are homogeneous coordinates of $C P_{i}^{1}$ in the configuration $(2.13)$ (e.g. $\mathscr{A}_{1}$ and $\mathscr{B}_{1}$ parametrize $C P_{1}^{1}$ ). The sub-determinants in Eq. (2.15) are now simply $\mathscr{A}_{2} \mathscr{B}_{1}, \mathscr{A}_{1} \mathscr{C}_{3}$, and $\mathscr{B}_{3} \mathscr{C}_{2}$, which do not vanish simultaneously, unless both of the coefficients of some of the equations Eq. (2.16) vanish. form:

6. $\widetilde{C P}_{p_{1}, \ldots, p_{4}}^{2} \in\left(\begin{array}{l|l}2 & 2 \\ 1 & 1\end{array}\right)_{7}=: \Gamma_{7}$. The quadric-linear constraint is of the general

$$
\mathscr{A}(2 \| 2) \cdot z_{0}+\mathscr{B}(2 \| 2) \cdot z_{1}=0, \quad\left(z_{0}, z_{1}\right) \in C P_{2}^{1} .
$$

In the four points where $\mathscr{A}=\mathscr{B}=0$ [since $\left.\chi_{E}(2 \| 22)=4\right]$, the coordinates $\left(z_{0}, z_{1}\right)$ are unconstrained by the above equation and span all of $C P_{2}^{1}$. Hence four (generically distinct) points of $C P_{1}^{2}$ are blown up. It is standard that two quadrics intersect at any four points which are in general position (in the sense of our Theorem 2). The converse now follows by construction.

7. $\widetilde{C P}_{p_{1}}^{2}, \ldots, p_{5} \in(4 \| 22)_{8}=: \Gamma_{8}($ see $[14$, p. 552$])$.

8. $\widetilde{C P_{p_{1}}^{2}, \ldots, p_{6}} \in(3 \| 3)_{9}=: \Gamma_{9}($ see $[14$, p. 489]).

Theorem 4. $\mathscr{S}$ is an iterated blow-up of $C P^{2}$ at 7 points in general position if and only if $\mathscr{S}$ is a double cover of $C P^{2}$ branched over a non-singular quartic. Moreover, the anti-canonical bundle of $\mathscr{S}$ is induced from the Hopf-bundle of $\mathrm{CP}^{2}$ by the covering map.

(For a proof we refer to pp. 545-549 of [14].)

Conjecture $Q$. Every non-singular homogeneous quartic on $C P^{2}$ is the sum of the squares of some three quadrics.

$\left.\begin{array}{l}\text { Proposition 9. If } \mathscr{S} \in\left(\begin{array}{l|l}2 \\ 1\end{array}\right. \\ \text { non-singular quartic. }\end{array}\right)_{10}$ then $\mathscr{S}$ is a double cover of $\widetilde{C P^{2}}$, branched over a Proof. The general form of the constraint is:

$$
\mathscr{A}(2 \| 2) \cdot z_{0}^{2}+\mathscr{B}(2 \| 2) \cdot z_{0} z_{1}+\mathscr{C}(2 \| 2) \cdot z_{1}^{2}=0, \quad\left(z_{0}, z_{1}\right) \in C P_{2}^{1} .
$$


This equation has two distinct solutions for $\left(z_{0}, z_{1}\right) \in C P_{2}^{1}$ except when the discriminant vanishes:

$$
\Delta^{2}=(\mathscr{B}(2 \| 2))^{2}-4(\mathscr{A}(2 \| 2) \cdot \mathscr{C}(2 \| 2))=0,
$$

in which case the solutions coincide. $\Delta^{2}$ is non-singular because the double cover is non-singular (see parenthetical remark on p. 548 of [14]).

Remark. If Conjecture $Q$ holds, so does the converse of Proposition 9:

$$
\widetilde{C P}_{p_{1}, \ldots, p_{7}}^{2} \in\left(\left.\begin{array}{l|l}
2 \\
1
\end{array}\right|_{2} ^{2}\right)_{10}:=\Gamma_{10} .
$$

We shall refer to the configurations $\Gamma_{3}, \Gamma_{4}^{0}, \Gamma_{4}^{1}, \Gamma_{5}, \ldots, \Gamma_{10}$ as special.

Theorem 5. If $\mathscr{S}$ belongs to a special configuration or is a double cover of $C P^{2}$ branched over a non-singular quartic, it is positive.

Proof. The statement is straightforward for cases with $\chi_{E} \leqq 9$, since these are represented by the special configuration given in the proof of Theorem 3, for which positiveness is manifest, since in each case the anti-canonical bundle is the restriction of a positive line bundle on the ambient space $\left(\mathscr{W}=\prod_{r=1}^{m_{\Gamma}} C P_{r}^{n_{r}}\right)$.

By Proposition 9 the case where $\left.\mathscr{S} \in\left(\begin{array}{l||l}2 \\ 1\end{array}\right)_{2}\right)_{10}$ is subsumed by the case in which $\mathscr{S}$ is a double cover of $C P^{2}$ branched over a non-singular quartic. Now by Theorem $4, \mathscr{K}_{\mathscr{S}}^{*}$ is induced from $\mathscr{H}_{C P^{2}}$ by the covering map. Since the Hopf bundle is positive, the Chern class of $\mathscr{K}_{\mathscr{S}}^{*}$ has a representative, $\Theta$, which is positive except at points of the branching curve where it is positive semi-definite and positive along the curve. Let $d^{2}$ be a smooth function on $\mathscr{S}$ which, near the branching set, is the square of the distance from the branching set in some smooth metric. Then $\partial \bar{\partial} d^{2}$ is exact and positive semi-definite along the branching set and positive in directions transverse to it. For sufficiently small $\varepsilon,\left(\Theta-\sqrt{-1} \varepsilon \partial \bar{\partial} d^{2}\right)$ is a positive representative of the Chern class of $\mathscr{K}_{\mathscr{S}}^{*}$.

Note that together, Theorems 2,3 , and 5 imply the equivalence of the following for all $\mathscr{S}$, except possibly when $\chi_{E}(\mathscr{S})=10$ :

1. $\mathscr{S}$ is a positive $\mathrm{SAR}$;

2. $\mathscr{S}=S_{0}$, or a successive blow-up of $C P^{2}$ at up to 7 points in general position;

3. $\mathscr{S}$ belongs to one of the special configurations.

Remark. If Conjecture $Q$ is true, the qualification for $\chi_{E}=10$ can be removed. In any case, $3 \Rightarrow 1 \Leftrightarrow 2$ without qualification.

\subsection{SARs with Euler Characteristic 12}

By Proposition 5, any SAR $Z$ with $\chi_{E}=12$ must be a nine-fold blow-up of $C P^{2}$ and by Corollary 3 it cannot be positive (this precludes the application of LHT).

Theorem 6. If a SAR $\mathscr{Z}$ is $C P^{2}$ blown up at nine points none of which is infinitely near another, it belongs to $\left(\begin{array}{l||l}2 & 3 \\ 1 & 1\end{array}\right)=: \Gamma_{12}$. 
Proof. The analysis analogous to that in 6. of the Proof of Theorem 3 shows that now nine distinct points, comprising the intersection of two cubics, are blown up [since $\left.\chi_{E}(2 \| 3 \quad 3)=9\right]$.

\subsection{Moduli Spaces}

Theorem 7. For each $n$ there exist spaces $\mathfrak{f}_{n}, \mathrm{~m}_{n}$ and a holomorphic submersion $\pi_{n}: \mathfrak{I}_{n} \rightarrow \mathfrak{m}_{n}$, such that:

1. $\operatorname{dim}\left(\mathrm{m}_{n}\right)=2 n$;

2. for each $\mu \in \mathfrak{m}_{n}, \pi_{n}^{-1}(\mu)$ is an $n$-fold iterated blow-up of $C P^{2}$;

3. every possible $\widetilde{C P_{p_{1}}^{2}, \ldots, p_{n}}$ occurs as $\mu$ ranges over $m_{n}$.

Remark. $\mathrm{m}_{n}$ is a considerably redundant moduli space for $n$-fold iterated blow-up of $C P^{2}$, not only SARs.

Proof. We prove this by induction, starting from $n=0$, where $\tilde{f}_{n}$ is $C P^{2}$ and $\mathrm{m}_{n}$ is a single point. Now, given $\tilde{f}_{n}$ and $\mathrm{m}_{n}$, set:

- $\mathrm{m}_{n+1}=\tilde{f}_{n}$;

- $\mathfrak{f}_{n+1}=\left\{(\varrho, \tau) \in \mathfrak{f}_{n} \times \mathfrak{f}_{n} \mid \pi_{n}(\varrho)=\pi_{n}(\tau)\right\}$ blown up along the diagonal. Finally we note that $\tilde{f}_{n+1}$ is fibred by the proper transform $\widetilde{\mathscr{M}}_{\tau}$ of the manifolds $\mathscr{M}_{\tau}=\left\{(\varrho, \tau) \mid \pi_{n}(\varrho)=\pi_{n}(\tau)\right\}$ for $\tau$ fixed, and that $\widetilde{\mathscr{M}}_{\tau}$ is $\pi_{n}^{-1}\left(\pi_{n}(\tau)\right)$ blown up at $\tau$.

A moduli space of surfaces with $\chi_{E}=12, n_{9}$, is obtained as the space of all twodimensional subspaces of the space of all cubics on $C P^{2}$.

We observe that for $i \leqq 7$ a dense open subset of the space $\mathrm{nt}_{i}$ corresponds to surfaces which are positive SARs. Similarly, a dense open subset of $n_{0}$ corresponds to SARs with $\chi_{E}=12$, satisfying the hypothesis, and hence the conclusion of Theorem 6.

In further support of the Conjecture that all SARs belong to configurations, we present the construction of a configuration that contains $S_{2}$ :

Consider the configuration:

$$
\left(\begin{array}{l||ll}
3 & 1 & 1 \\
1 & 1 & 1
\end{array}\right)=: \Gamma_{4}^{+}
$$

the two bi-linear constraints in $\Gamma^{\prime}$ are of the form:

$$
\mathscr{A} z_{0}+\mathscr{B} z_{1}=0, \quad \mathscr{C} z_{0}+\mathscr{D} z_{1}=0,
$$

where $\left(z_{0}, z_{1}\right) \in C P^{1}$ and the "coefficients" $\mathscr{A}-\mathscr{D}$ are linear in the homogeneous coordinates of $C P^{3}$ and thus correspond to planes. In order for the above system of equations to have a non-trivial solution $\left[\right.$ since $\left.(0,0) \notin C P^{1}\right]$ the determinant:

$$
\operatorname{det}\left(\begin{array}{cc}
\mathscr{A} & \mathscr{B} \\
\mathscr{C} & \mathscr{D}
\end{array}\right)=0
$$

must be satisfied, so the matrix must be of rank 0 or 1 . However, for it to be of rank 0 , all four "coefficients" ought to vanish which does not happen (four planes do not meet) generically in $C P^{3}$.

Therefore, in the generic case, the two constraints of the configuration $\Gamma_{4}^{+}$may be replaced by the constraint in Eq. (2.23) imposed on $C P^{3}$ only. This then 
becomes $(3 \| 2)_{4} \ni S_{0}$, and it can be shown [14] that any non-singular quadric in $C P^{3}$ can be represented by Eq. (2.23).

If however the matrix in Eq. (2.23) is indeed of rank 0 at some point in $C P^{3}$, the configuration $\Gamma_{4}^{+}$represents an $S_{2}$ surface, which is not represented by the $(3 \| 2)_{4}$ configuration. This result should be compared with the procedure of [9]; in particular, their "reduction rule":

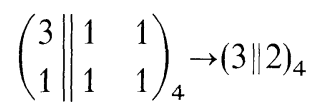

dismisses any Calabi-Yau manifolds that can be embedded in $S_{2} \times \mathscr{W}$ but not in $S_{0} \times \mathscr{W}$ (where $\mathscr{W}$ denotes a product of some complex projective spaces).

Also, note that the configuration (2.13), when the matrix in Eq. (2.14) is of rank 1 at some point in $C P^{1} \times C P^{1} \times C P^{1}$, corresponds to surfaces with exceptional lines of the $2^{\text {nd }}$ kind, which are not positive.

Similarly to the augmentation of $\Gamma_{6}$ to configuration (2.13), one can augment any of the special configurations $\Gamma_{3}, \ldots, \Gamma_{10}$ and $\Gamma_{12}$; this procedure was termed "splitting" by Candelas et al. in [9]. Unlike the special ones, the "split" configurations generally contain surfaces that have exceptional lines of the $2^{\text {nd }}$ kind; hence many non-ample SARs belong to configurations.

The fact that surfaces having exceptional lines of the $2^{\text {nd }}$ kind are not contained in the simple configurations, but (at least some of them) are in "split" configurations should be taken into account and treated with special care in any final classification of CICY.

Remark. While $S_{0}$ is an infinitesimal deformation of $S_{2}, S_{1}$ is not a deformation of either and is, in fact, of a distinct homotopy type.

\section{Hypersurfaces in Products of SARs}

Having discussed the SARs, we are now ready to consider the Calabi-Yau manifolds embedded as hypersurfaces in product of SARs [see Eq. (2.3)].

If $\mathscr{S}$ and $\mathscr{S}^{\prime}$ are SARs (not assumed to belong to configurations), we extend the

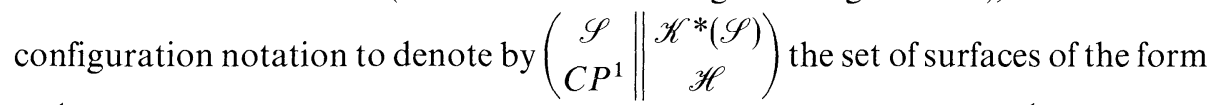
$\chi^{-1}(0)$, where $\chi$ is a section of the bundle $\mathscr{K}^{*} \otimes \mathscr{H}$ over $\mathscr{S} \times C P^{1}$ with nonvanishing one-jet. Similarly, we write $\left(\begin{array}{c}\mathscr{S} \\ \mathscr{S}^{\prime}\end{array} \| \begin{array}{c}\mathscr{K}^{*}(\mathscr{S}) \\ \mathscr{K}^{*}\left(\mathscr{S}^{\prime}\right)\end{array}\right)$ to denote the set of CalabiYau 3 -folds of the form $\varphi^{-1}(0)$ where $\varphi$ is a section of $\mathscr{K}_{\mathscr{S}}^{*} \times \mathscr{S}^{\prime}=\mathscr{K}_{\mathscr{S}}^{*} \otimes \mathscr{K}_{\mathscr{S}^{\prime}}^{*}$ with nowhere vanishing one-jet.

Proposition 10. Let $\Gamma$ and $\Gamma^{\prime}$ be positive SARs with $3 \leqq \chi_{E} \leqq 10$. Then:

$$
\begin{gathered}
\chi_{E}\left(\begin{array}{c}
\mathscr{S} \\
\mathscr{S}
\end{array} \| \begin{array}{c}
\mathscr{K}_{\mathscr{S}}^{*} \\
\mathscr{K}_{\mathscr{S}^{\prime}}^{*}
\end{array}\right)=-2 \cdot\left(12-\chi_{E}(\mathscr{S})\right) \cdot\left(12-\chi_{E}\left(\mathscr{S}^{\prime}\right)\right), \\
b_{1,1}\left(\begin{array}{c}
\mathscr{S} \\
\mathscr{S}^{\prime}
\end{array} \| \begin{array}{c}
\mathscr{K}_{\mathscr{S}}^{*} \\
\mathscr{K}_{\mathscr{S}^{\prime}}^{*}
\end{array}\right)=b_{1,1}(\mathscr{S})+b_{1,1}\left(\mathscr{S}^{\prime}\right) .
\end{gathered}
$$

Proof. By direct computation for $\chi_{E}$, and by LHT for $b_{1,1}$. 
Theorem 8. The hypothesis of positivity can be removed from Proposition 10.

Proof. Let $\mathscr{S}_{1}$ and $\mathscr{S}_{1}$ be SARs with $\chi_{E}\left(\mathscr{S}_{1}\right), \chi_{E}\left(\mathscr{S}_{2}\right) \leqq 10$. Set $m=\chi_{E}\left(\mathscr{S}_{1}\right)-3$, $n=\chi_{E}\left(\mathscr{S}_{2}\right)-3$. We write $\pi$ for

$$
\pi_{m} \times \pi_{n}: \tilde{\mathfrak{f}}_{m} \times \tilde{\mathfrak{T}}_{n} \rightarrow \mathrm{m}_{m} \times \mathrm{m}_{n} .
$$

Then we can identify $\mathscr{S}_{1} \times \mathscr{S}_{2}$ with $\pi^{-1}(p)$ for some $p \in \mathrm{m}_{m} \times \mathrm{m}_{n}$. Let $\xi$ be a section of $\mathscr{K}_{\mathscr{S}_{1} \times \mathscr{I}_{2}}^{*}=\mathscr{K}_{\mathscr{S}_{1}}^{*} \otimes \mathscr{K}_{\mathscr{S}_{2}}^{*}$ with nowhere vanishing one-jet.

We may identify $\mathscr{K}_{\mathscr{S}_{1} \times \mathscr{S}_{2}}^{*}$ with $\left.\mathscr{K}^{*}\left(\mathfrak{f}_{m} \times \tilde{\mathfrak{f}}_{n}\right)\right|_{\pi^{-1}(p)}$. Then by the Griffiths obstruction theory [16], the obstruction to extending $\xi$ to a section of $\mathscr{K} *\left(\mathfrak{f}_{m} \times \tilde{f}_{n}\right)$ over a neighborhood of $\pi^{-1}$ lie in

$$
H^{1}\left(\mathscr{T}_{1} \times \mathscr{S}_{2}, \mathscr{K}_{\mathscr{S}_{1} \times \mathscr{S}_{2}}^{*} \otimes \mathbf{S}^{n}\left(\mathscr{N}_{p}\right)\right)
$$

where $\left.\mathbf{S}^{n}\left(\mathscr{N}_{p}\right)\right)$ denotes symmetric $n$-fold products of the normal bundle to $\pi^{-1}(p)$. Since $\mathscr{N}_{p}$ is trivial, by Proposition 8 and the Künneth formula, all these groups vanish.

Let $\tilde{\xi}$ be an extension of $\xi$ to a neighborhood of $\pi^{-1}(p)$. Then, since $\pi$ is open and $\pi^{-1}(p)$ is compact, the domain of $\widetilde{\xi}$ includes $\pi^{-1}(\mathscr{U})$ for some neighborhood $\mathscr{U}$ of $p$. Moreover, by making $\mathscr{U}$ sufficiently small, we can ensure that $\widetilde{\xi}_{(}\left(\pi^{-1}(\mathscr{U})\right)$ has nowhere vanishing one-jet along the fibres of $\pi$. It follows that the Calabi-Yau manifolds $\xi^{-1}(0) \cap \pi^{-1}(q)$ are deformations of one another for $q \in \mathscr{U}$. The result now follows from the positivity of $\pi^{-1}(q)$ for a dense open subset of $m_{m} \times m_{n}$.

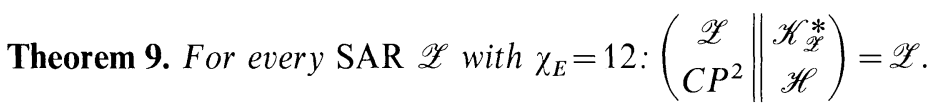

Proof. By Proposition 3, the anti-canonical bundle of $\mathscr{Z}$ is induced by a map $f: \mathscr{Z} \rightarrow C P^{1}$ (since there are only two sections), while $\mathscr{H}_{1}$ is induced by the identity map:

$$
\begin{array}{rrr}
\mathscr{Z} & \times C P^{1} \\
\downarrow^{f} & \downarrow^{i d} \\
C P_{\mathscr{Z}}^{1} & \times C P^{1}
\end{array} .
$$

Denoting $\mathscr{F}=(f$, Id $)$, and by $\mathscr{H}_{\mathscr{Z}}$ and $\mathscr{H}_{1}$ the Hopf-bundles of $\mathscr{Z}$ and $C P^{1}$ respectively, one has that $\mathscr{F}^{*}\left(\mathscr{H}_{\mathscr{Z}}, \mathscr{H}_{1}\right)=\mathscr{K}_{\mathscr{Z}}^{*} \otimes \mathscr{H}_{1}$. Given a section $\sigma$ of $\mathscr{H}_{\mathscr{Z}} \otimes \mathscr{H}_{1}$, one defines $\chi=\mathscr{F}^{*}(\sigma)$, so that $\chi^{-1}(0)=\mathscr{F}^{-1}\left(\sigma^{-1}(0)\right)$. Without loss of generality, $C P_{\mathscr{Z}}^{1}$ and $C P^{1}$ can be identified, so that $\sigma^{-1}(0)=\operatorname{diag}\left(C P_{\mathscr{Z}}^{1} \times C P^{1}\right)$, whereby:

$$
\mathscr{F}^{-1}\left(\sigma^{-1}(0)\right)=\{(s, t) \mid f(s)=t\}=\{(s, f(s))\} \approx \mathscr{Z},
$$

with $s$ denoting the points of $\mathscr{Z}$ and $t$ the points of $C P^{1}$.

Proposition 11. For any SAR $\mathscr{S}$ and a SAR $\mathscr{Z}$ with $\chi_{E}(\mathscr{Z})=12$, there is a SAR $\mathscr{Z}^{\prime}$ with $\chi_{E}=12$ such that:

$$
\left(\begin{array}{c||c}
\mathscr{S} & \mathscr{K}_{\mathscr{S}}^{*} \\
\mathscr{Z} & \mathscr{K}_{\mathscr{X}}^{*}
\end{array}\right)=\left(\begin{array}{c||c}
\mathscr{Z} \\
\mathscr{Z}^{\prime}
\end{array} \mid \begin{array}{c}
\mathscr{K}_{\mathscr{X}}^{*} \\
\mathscr{K}_{\mathscr{Z}^{\prime}}^{*}
\end{array}\right)
$$


Proof. By Theorem 9,

$$
\mathscr{Z}=\left(\begin{array}{c||c}
\mathscr{Z} & \mathscr{K}_{\mathscr{Z}}^{*} \\
C P^{1} & \mathscr{H}_{1}
\end{array}\right) .
$$

On the other hand, straightforward computation yields:

$$
\begin{aligned}
\chi_{E}\left(\begin{array}{c}
\mathscr{S} \\
C P^{1} \| \\
\mathscr{K}^{*}(\mathscr{S}) \\
\mathscr{H}
\end{array}\right) & =\frac{1}{2} \underset{\left(\mathscr{S} \times C P^{1}\right)}{\int_{1}}\left(c_{1}^{2}(\mathscr{S})+c_{2}(\mathscr{S})\right) c_{1}\left(C P^{1}\right) \\
& =C_{1}^{2}(\mathscr{S})+C_{2}(\mathscr{S}) \\
& =12, \quad \text { by Eq. }(2.8),
\end{aligned}
$$

and by Proposition 5, this configuration must be a nine-fold iterated blow-up of $C P^{2}$. Combining these two:

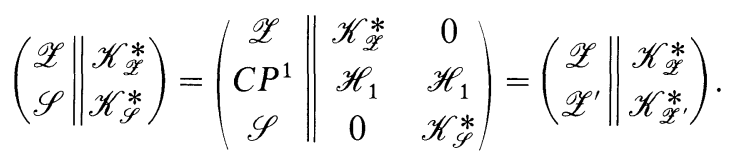

Since the $\chi_{E}=12$ SARs are not positive, LHT is not applicable to the Calabi-Yau manifolds considered in Proposition 11; the spectral sequence method [13] yields only the bounds $19 \leqq b_{1,1} \leqq 23$. However, C. Schoen has studied a class of possibly singular varieties [17] which includes these Calabi-Yau manifolds in the nonsingular case. It follows from his formula (7.4) that $b_{1,1}=19$.

Using the results of the present paper together with those of $[8,13]$ and Candelas et al. in [9], the Hodge diamond has recently been explicitly computed by A. Lütken for all Calabi-Yau manifolds which can be realized as complete intersections in products of complex projective spaces.

Acknowledgements. We would like to thank P. Candelas and M. Cornalba for some very helpful discussions. This work has been supported in part by the National Science Foundation.

\section{References}

1. Schwarz, J.H.: Superstrings - a collection of reprints - Singapore: World Scientific 1985 Green, M.B., Schwarz, J.H., Witten, E.: Superstring theory, Vol. I and II. Cambridge: Cambridge University Press 1987

2. Candelas, P., Horowitz, G.T., Strominger, A., Witten, E.: Vacuum configurations for superstrings. Nucl. Phys. B 258, 46 (1985)

3. Witten, E.: Symmetry breaking patterns in superstring models. Nucl. Phys. B 258, 75 (1985)

4. Calabi, E.: In: Algebraic geometry and topology: A symposium in honous of S. Lefschetz. Princeton, NJ: Princeton University Press 1957, pp. 78

Yau, S.-T.: Proc. Natl. Acad. Sci. 74, 1798 (1977)

5. Yau, S.-T.: In: Proc. of Symposium on anomalies, geometry, topology, Bardeen, W.A., White, A.R. (eds.). Singapore: World Scientific 1985

6. Strominger, A., Witten, E.: New manifolds for superstring compactification. Commun. Math. Phys. 101, 341 (1985)

7. Hübsch, T.: Calabi-Yau manifolds - Motivations and constructions. Commun. Math. Phys. 108, 291 (1987)

Manifold compactification of superstrings. In: the Proceedings of the Summer Workshop on High Energy Physics and Cosmology, ICTP, Trieste, Italy (1986) 
8. Green, P., Hübsch, T.: Calabi-Yau manifolds as complete intersections in products of complex projective spaces. Commun. Math. Phys. 109, 99 (1987)

9. Aspinwall, P.S., Greene, B.R., Kirklin, K.H., Miron, P.J.: Searching for three-generation Calabi-Yau manifolds. University of Oxford Report February 1987

Candelas, P., Dale, A.M., Lütken, C.A., Schimmrigk, R.: Complete intersection Calabi-Yau manifolds. University of Texas Report UTTG-10-87

10. Bott, R.: On a theorem of Lefschetz. Mich. Math. J. 6, 211 (1959)

11. Greene, B.R., Kirklin, K.H., Miron, P.J., Ross, G.G.: A three generation superstring model I. Nucl. Phys. B 278, 667 (1986); A superstring inspired standard model. Phys. Lett. 180B, 69 (1986)

Goodman, M.W., Witten, W.: Global symmetries in four and higher dimensions. Nucl. Phys. B 271, 21 (1986)

Kalara, S., Mohapatra, R.N.: $C P$ violation and Yukawa couplings in superstring models. University of Minnesota Report UMN-TH-590-86, Phenomenology of a Four Generation Superstring Model. University of Maryland Report PP-87-123

12. Kodaira, K.: Complex manifolds and deformation of complex structures. Berlin, Heidelberg, New York: Springer 1986

13. Green, P., Hübsch, T.: Polynomial deformations and cohomology of Calabi-Yau manifolds. Commun. Math. Phys. 113, 505-528 (1987)

14. Griffiths, P., Harris, J.: Principles of algebraic geometry. New York: Wiley 1978

15. Hirzebruch, F.: Topological methods in algebraic geometry. Berlin, Heidelberg, New York: Springer 1978

16. Griffiths, P.: The extension problem for compact submanifolds of complex manifolds. I. In: Proc. of Conference on Complex Analysis, pp. 113-142. Minneapolis, 1964. Berlin, Heidelberg, New York: Springer 1965

17. Schoen, C.: On fiber products of rational elliptic surfaces with section. Mathematical Sciences Research Institute Report MSRI 04620-87, and private communication

Communicated by S.-T. Yau

Received June 10, 1987; in revised form October 5, 1987 